\title{
Probing polyelectrolyte adsorption in charged nanochannels by streaming potential measurements
}

\author{
Anastasia Christoulaki ${ }^{1}$, Didier Lairez ${ }^{2}$, Emmanuelle Dubois ${ }^{1}$ and Nicolas Jouault ${ }^{*}$ \\ 1 Sorbonne Université, CNRS, Laboratoire PHENIX, F-75005, Paris, France \\ ${ }^{2}$ Laboratoire des Solides Irradiés (LSI), École polytechnique, CNRS, CEA, Institut Polytechnique de Paris, 91128 Palai- \\ seau cedex, France
}

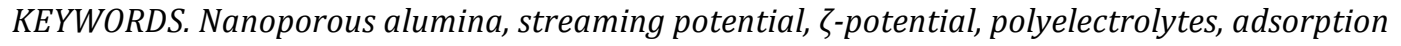

\begin{abstract}
It remains a great experimental challenge to obtain quantitative information on the polyelectrolyte (PE) behavior confined in charged nanoporous materials. Here we propose an original approach using transverse streaming potential measurements (TSPMs), an efficient technique providing information on the electrical surface properties of nanoporous materials through the $\zeta$-potential determination. We conduct TSPMs within the thin double-layer approximation on a model system composed of individual nanochannels, nanoporous anodic aluminum oxide (AA0) membrane, filled with a wellknown PE, sodium polystyrene sulfonate (NaPSS). We demonstrate that TSPM can provide the AAO $\zeta$-potential under different experimental conditions and monitor the PE penetration in AAO with positive or negative surface charge. On positive surface, the PE irreversibly adsorbs, while it doesn't when the surface is negatively charged, indicating the electrostatic nature of the PE adsorption. In a context of experimental limitations to investigate PE behavior on concave surfaces, this study shows that TSPM is suitable to extract quantitative information and can be exploited to gain understanding on the PE adsorption and desorption $\quad$ in $\quad$ confined
\end{abstract}

Polyelectrolytes (PEs) are polymers carrying electric charges due to the dissociation in polar solvents of ionic groups present on the chain ${ }^{1}$. The charges have direct consequences on the PEs behavior in solution compared to neutral polymers ${ }^{2}$. When a PE is confined in nanoporous materials, its properties (conformation, dynamics, transport or adsorption) are affected by the geometrical or electrostatic constraints imposed by the confining medi$\mathrm{um}^{3}$. The better understanding of these modifications is relevant for many potential applications such as ionic separation $^{4}$, catalysis ${ }^{5}$ or ionic rectification for single molecules analysis ${ }^{6}$. However, few experimental studies ${ }^{7-11}$ investigated the effects of such confinement on the PEs characteristics, presumably due to experimental difficulties to collect quantitative information.

In particular, when dealing with charged interfaces, the surface charge of the nanoporous material plays a critical role and its determination is crucial for further experimental investigations involving PEs. The classical techniques used to characterize surface charges of nanoparticles in solution (such as electrophoretic light scattering ${ }^{12}$ or acid/base titration ${ }^{13}$ ) cannot be applied for porous materials and alternative approaches have to be found. For instance, indirect information on the charge can be obtained by the titration of radioactive elements after diffusion and adsorption in the porous medium: if positive ions adsorb then the surface is negatively charged ${ }^{14}$. However, to have more quantitative information on the charge, experiments based on electrokinetic phenomena as electro- osmosis or streaming potential measurements (SPMs) to determine the $\zeta$-potential have to be used ${ }^{15-19}$. But, so far, a very limited number of experimental studies really dealt with such characterization, bringing out the challenging nature of experiments in nanoporous media.

Here, we address these issues by presenting an original approach using SPM on a model nanoporous system, namely nanoporous anodic aluminum oxide (AAOs) membranes, composed of parallel and non-connected nanochannels. We show that SPM is an efficient technique to access the AAO electrical surface properties under different experimental conditions and to further monitor the PE diffusion in AAOs with positive or negative surface charge.

The AAO membranes are prepared by the classical twostep electrochemical anodization of high purity aluminum foil (Al, 99.999\% from Goodfellow, $320 \mu \mathrm{m}$ thick). A first anodization is performed in $0.3 \mathrm{M}$ oxalic acid solution constantly stirred under continuously applied voltage of $40 \mathrm{~V}$ at $\mathrm{T}=18^{\circ} \mathrm{C}$. After two hours, both sides of the Al foil present the formed oxide, which is subsequently dissolved in an aqueous solution of phosphochromic acid (6 wt.\% $\mathrm{H}_{3} \mathrm{PO}_{4}$ and 1.8 wt. $\% \mathrm{CrO}_{3}$ ) during 2 hours at $\mathrm{T}=50^{\circ} \mathrm{C}$. Then, a second anodization is performed for 8 hours under the same conditions as the first one. Finally, the AAO membrane is obtained by the detachment of the oxide from the remaining $\mathrm{Al}$ through a third anodization step made in 12 $\mathrm{M} \mathrm{H}_{2} \mathrm{SO}_{4}$ under $40 \mathrm{~V}$ and $\mathrm{T}=0^{\circ} \mathrm{C}$ for 7 minutes followed by an immersion in phosphochromic acid during $30 \mathrm{~min}$ at $30^{\circ} \mathrm{C}^{20}$. During this last step the alumina is easily detached 
from both sides of the Al substrate and two through-hole membranes are obtained.

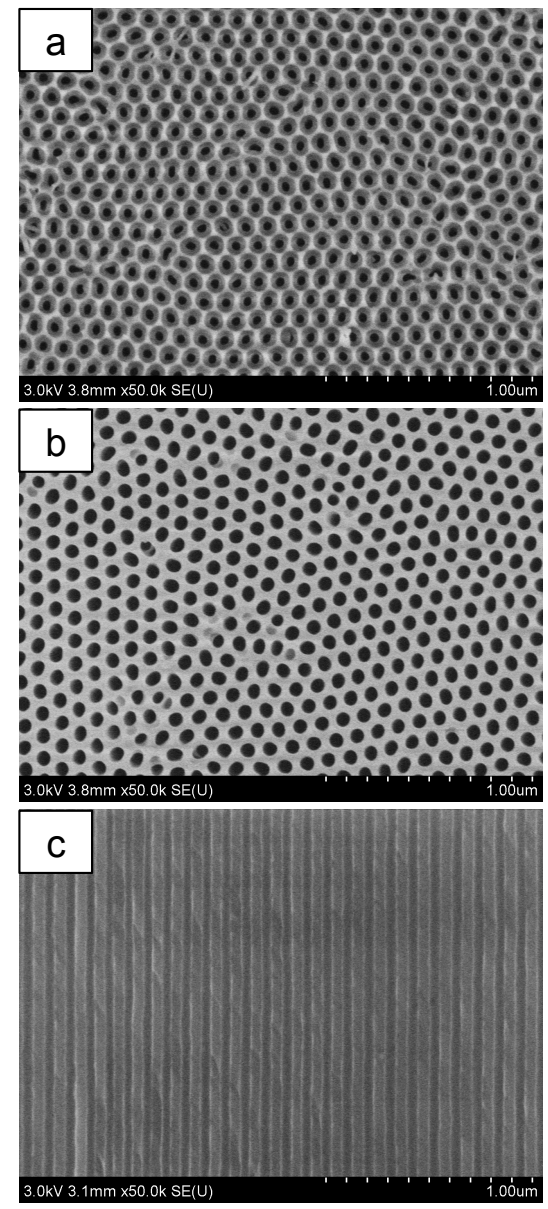

Figure 1. SEM image of a detached AAO membrane prepared in $0.3 \mathrm{M}$ oxalic acid under $40 \mathrm{~V}$ : a) top surface, b) bottom surface, c) section view. The AAO total thickness is around 62 $\mu \mathrm{m}$. Scale bar: $1 \mu \mathrm{m}$.

Fig. 1 shows Scanning Electron Microscopy images (SEM, using a Hitachi SU-70 FEGSEM under an accelerating voltage of 1 or $3 \mathrm{kV}$ ) for a typical AAO membrane prepared under the conditions detailed above. From the image analysis, one obtains a pore diameter $D_{p}$ of $40+/ 1 \mathrm{~nm}$ for the top surface (Fig. 1a) while the bottom $D_{p}$ was systematically larger with a value of $66+/-3 \mathrm{~nm}$ (Fig. 1b). This difference is due to the high dissolution of alumina produced in $12 \mathrm{M} \mathrm{H}_{2} \mathrm{SO}_{4}$ during the $3^{\text {rd }}$ anodization that enlarges the bottom pore. SEM images from the section view show that the nanochannels are straight and not interconnected (Fig. 1c) and adopt a bilayer structure composed of a thick layer of $\approx 58 \mu \mathrm{m}$ with a $\mathrm{D}_{\mathrm{p}}$ of $42 \mathrm{~nm}$ and a thin layer of $\approx 4 \mu \mathrm{m}$ with a $D_{p}$ of $66 \mathrm{~nm}$ (see Fig. S1). This residual thin layer comes from the $3^{\text {rd }}$ anodization and has a negligible contribution to the TSPM ${ }^{21}$, also confirmed by complementary water flow measurements (see details in SI) through the $\mathrm{AAO}^{22}$ giving a hydrodynamic diameter of $47 \mathrm{~nm}$, close to the top surface $D_{p}$. Finally, since the aspect ratio is greater than 1000, the AAOs can be considered as a collection of infinitely long nanochannels. Note that, for this work, we synthesized several AAOs under the same conditions to perform all SPM measurements and the average $D_{p}$ values are $38+/ 5 \mathrm{~nm}$ and $62+/-4 \mathrm{~nm}$ for the top and bottom surface, respectively, indicating the good reproducibility of the AAO synthesis.

Then, SPMs have been performed to quantify the AAO electrical surface properties. In contact with water, the pore-wall-surface of aluminum oxide undergoes chemical reactions producing metal-hydroxyl groups whose protonation and deprotonation is responsible for the charge of the surface. Because of electroneutrality, these static charges are compensated by an imbalance in the amount of anions and cations in solution. Thus, the flow of an electrolyte solution through an AAO membrane caused by a difference of pressure $\Delta \mathrm{P}$ on its two sides produces an electric current (namely the streaming current) that comes with a difference of electric potential between the two sides (namely the streaming potential $U_{\text {str }}{ }^{23}$. The measurement of the variation of $U_{\text {str }}$ with the pressure difference gives directly access to the $\zeta$-potential, which is defined as the electric potential at a distance from the porewall-surface at which the liquid flow velocity is zero and is given by the following expression:

$$
\zeta=\frac{\sigma \eta}{\varepsilon} \frac{d U_{s t r}}{d P}
$$

where $\sigma$ is the electrolyte conductivity, $\eta$ is the viscosity and $\varepsilon$ the dielectric permittivity. Eq. (1) is obtained by neglecting the contribution of surface conductivity of the electric double-layer to the pore conductance (for discussion and details on this point see the SI). This assumption is also confirmed by the literature where the surface conductance is typically neglected for $\kappa R_{p}>10$ and low zeta potential value ${ }^{24}$ (here we use as electrolyte $50 \mathrm{mM} \mathrm{KCl}$, with a Debye length $\kappa^{-1}$ of $1.4 \mathrm{~nm}$ at $298 \mathrm{~K}$ and a $\kappa \mathrm{R}_{\mathrm{p}} \approx 11.5$, indicating the non-overlapping of the Debye length).

Previous SPMs on AAOs have been performed by imposing a flow along the surface of the membrane (tangential SPM) ${ }^{18}$ or through the membrane (transverse SPM or TSPM) ${ }^{16,17,25}$, the latter being only used so far for large AAO pore sizes (around 0.1 and $0.2 \mu \mathrm{m}$ ). Although TSPM is mainly sensitive to the internal nanochannel surface, which is by far the largest, it has not been used to probe the PE behavior inside nanochannels; an approach we want to address. Here, TSPMs are performed with a homemade device consisting in two compartments separated by the $\mathrm{AAO}$ membrane and containing the electrolyte and one $\mathrm{Ag} / \mathrm{Ag}-\mathrm{Cl}$ electrode each. Electric measurements are carried out with the electro-physiology amplifier Axopatch $200 \mathrm{~B}$ used in "I=0" mode. The pressure difference is measured with a piezoelectric differential sensor and obtained by means of air compressed by a syringe pump applied to one of the compartments, while the other is left at atmospheric pressure. Different buffer solutions with $\mathrm{pH}$ ranging from 3.6 to 10.7 (listed in Table S3) were prepared and diluted with ultrapure water to reach a concentration of the buffer of $2 \mathrm{mM}$. $\mathrm{KCl}$ was then added at a fixed concentration of $50 \mathrm{mM}$ to set the solution's conductivity to a constant value of $0.68 \mathrm{~S} / \mathrm{m}$. Note that the AAO stability in water has been checked with time and the $\zeta$-potential value remains constant over a period of $16 \mathrm{~h}$ (time larger than our longest measurement time), indicating that the possi- 
ble AAO hydration has no influence on the $\zeta$-potential (Fig. S5).

Fig. 2 shows the evolution of the AAO $\zeta$-potential as a function of $\mathrm{pH}$. In acidic conditions $(\mathrm{pH}<5.5)$ the $\zeta$ potential is positive while it is negative in basic conditions $(\mathrm{pH}>9)$ with a sign inversion (or isoelectric point IEP) around a $\mathrm{pH}$ of 8.5 . On the contrary, in neutral conditions $(5.5<\mathrm{pH}<8)$, the sign of the $\zeta$-potential depends on the nature of the buffer molecules: the value is always positive (acetic acid/acetate $\left(\mathrm{N}^{\circ} 1\right.$ to 5$)$, ammonia/ammonium $\left(\mathrm{N}^{\circ}\right.$ 7) or $\left.\mathrm{KOH}\left(\mathrm{N}^{\circ} 8\right)\right)$, except for the phosphate buffers where it is negative $\left(\mathrm{N}^{\circ} 15\right.$ to 18$)$.

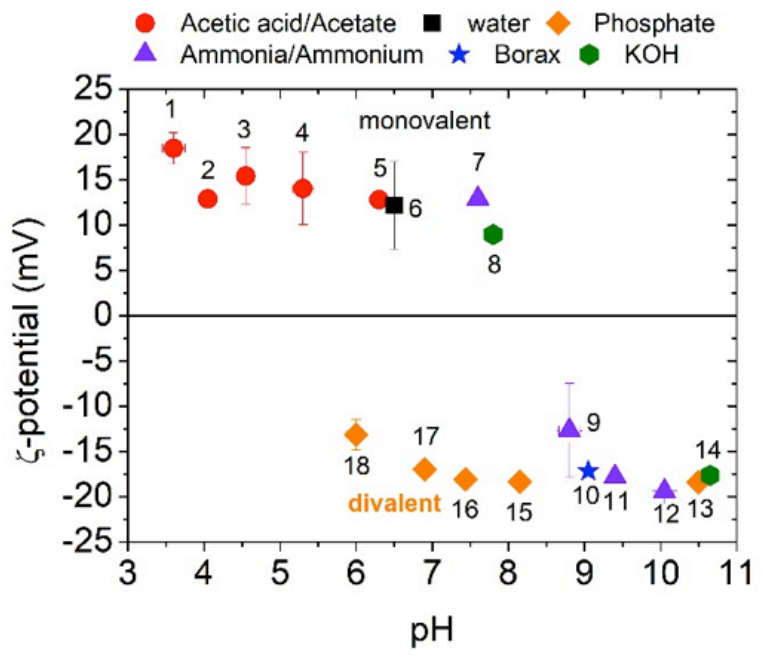

Figure 2. $\zeta$-potential of AAO membranes as a function of the $\mathrm{pH}$ in different buffer solutions (the $\mathrm{N}^{\circ}$ correspond to the $\mathrm{N}^{\circ}$ listed in Table S1). The reported values are obtained at equilibrium and the error bars correspond to the standard deviation of different measurements done on different samples.

The negative $\zeta$-potential obtained when tuning the $\mathrm{pH}$ with phosphate buffers must be attributed to specific adsorption of phosphate ions and more specifically to divalent $\mathrm{HPO}_{4}{ }^{2-}$, which is the dominant species in this $\mathrm{pH}$ region $\left(\mathrm{pKa}_{1}=2.12, \mathrm{pKa}_{2}=7.2\right)$. Indeed, divalent and trivalent ions have been reported to adsorb strongly on the surfaces and shift the IEP of charged membranes ${ }^{19}$. Moreover, the phosphate adsorption has a slow kinetics as shown in Fig. S4a (around $15 \mathrm{~min}$ to reach a constant negative value of $10 \mathrm{mV}$ ) and phosphate ions can be desorbed by washing the AAO with acetic acid/acetate buffer at pH=4.6 (see Fig. S4b).

From the literature, if there is no specific ion adsorption, the point of zero charge (PZC) or the isoelectric point (IEP) vary from $\mathrm{pH}=7.5$ to 9.5 for alumina nanoparticles (NPs) ${ }^{26}$ and from $\mathrm{pH}=4$ to 7 for flat surface ${ }^{27}$. This wide range of $\mathrm{PZC} / \mathrm{IEP}$ is mainly due to variations in the hydroxide coordination that depends on the crystal structure and composition $^{27}$. Here, our measured IEP $(\mathrm{pH}=8.5)$ is similar to NPs and to previous AAO membrane prepared in $0.22 \mathrm{M}$ oxalic acid for which the IEP measured by tangential SPMs varies from $\mathrm{pH}=7.8$ to 6.7 when the pore diameter decreases from $40 \mathrm{~nm}$ to $15 \mathrm{~nm}^{18}$
Finally, this ion specific adsorption is a very convenient way to tune negatively the AAO charges to avoid working under basic conditions (where fast AAO dissolution occurs) or with grafting molecules whose charge is $\mathrm{pH}$ dependent. This strategy will be exploited in the following to study the PE behavior in AAO with positive or negative surface charge under neutral $\mathrm{pH}$ conditions.

We used the widely studied sodium polystyrene sulfonate $^{1}$ as the PE (NaPSS purchased from Sigma Aldrich, $\mathrm{M}_{\mathrm{w}}=70 \mathrm{kDa}, \mathrm{PDI}=1.8$ ), which is a strong PE carrying one charged group per monomer. In-situ TSPMs have been conducted in such conditions that the PE diffusion is favored, i.e. $D_{H}<D_{P}$ (where $D_{H}=20 \mathrm{~nm}$ is the hydrodynamic $\mathrm{PE}$ diameter) and working at low $\mathrm{PE}$ concentration $\mathrm{C}_{\mathrm{PE}}$, i.e. in the dilute regime $\left(\mathrm{C}_{\mathrm{PE}}<\mathrm{C}^{*}\right.$, where the overlap concentration $\mathrm{C}^{*}$ estimated of the order of $26 \mathrm{~g} / \mathrm{L}$ from specific viscosity measurements). As mentioned above, we work at neutral $\mathrm{pH}(\mathrm{pH}=6.5)$ where the AAO surface can be positive in pure water or negative in phosphate buffer solution. Note that, as for TSPMs measurements in empty AAOs, $\mathrm{KCl}$ is added in the PE solution at a concentration of $50 \mathrm{mM}$ to keep the conductivity at a constant value.

We will start with the case of positive AAO. Fig. 3 shows the $\zeta$-potential evolution with time of an AAO immersed in NaPSS aqueous solution at a concentration of $0.14 \mathrm{~g} / \mathrm{L}$. At $\mathrm{t}=0$, the AAO is positively charged ( $\zeta$-potential around 25 $\mathrm{mV}$ ) and gradually reverses from positive to negative, until the $\zeta$-potential stabilizes at a constant value of $-23 \mathrm{mV}$, indicating the gradual adsorption of PE chains at the surface until the equilibrium is reached. This constant $\zeta-$ potential evolution over a long time (more than $1000 \mathrm{~min}$ ) is consistent with previous works indicating that, at low salt concentration $(<0.1 \mathrm{M})$, the first adsorbed PE chains remains strongly adsorbed and are not displaced by other chains ${ }^{28}$, suggesting that there is no chain reorganizations with time. The time evolution of the $\zeta$-potential can be fitted with a simple exponential to extract a characteristic time of adsorption $\tau_{\mathrm{C}}$ of around $300 \mathrm{~min}$, suggesting a slow adsorption process. The fitting with one simple exponential supposes that PE adsorption on the external top and bottom AAO surfaces doesn't influence the PE entering process into the nanochannels. Immediately after the PE adsorption, in order to evaluate the strength of the PE adsorption, the TSPM cell was filled with acetic acid/acetate buffer $(\mathrm{pH}=3)$ and the $\zeta$-potential is measured over a given time (red squares in Fig. 3). The $\zeta$-potential remains negative around $-15 \mathrm{mV}$, indicating that the adsorbed PE chains are not washed away. The increase from $-23 \mathrm{mV}$ to $-15 \mathrm{mV}$ suggests either some desorption of loosely adsorbed chains or modification of the conformation of the adsorbed layer. Note that the adsorbed chains cannot be removed after a sequence of washing in neutral/basic/acid/neutral buffers indicating the irreversibility of the PE adsorption (see Fig. S6). The hydrodynamic PE layer thickness is estimated to be around $1 \mathrm{~nm}$ by flow measurements (see Fig. S2). The PSS covered nanochannel surface can still be approximated as an impermeable hard surface from which eq. 1 is still valid. For thicker soft permeable layer, appropriate electrohydrodynamic theories have to be used to correctly interpret streaming potential measurements ${ }^{29,30}$. 


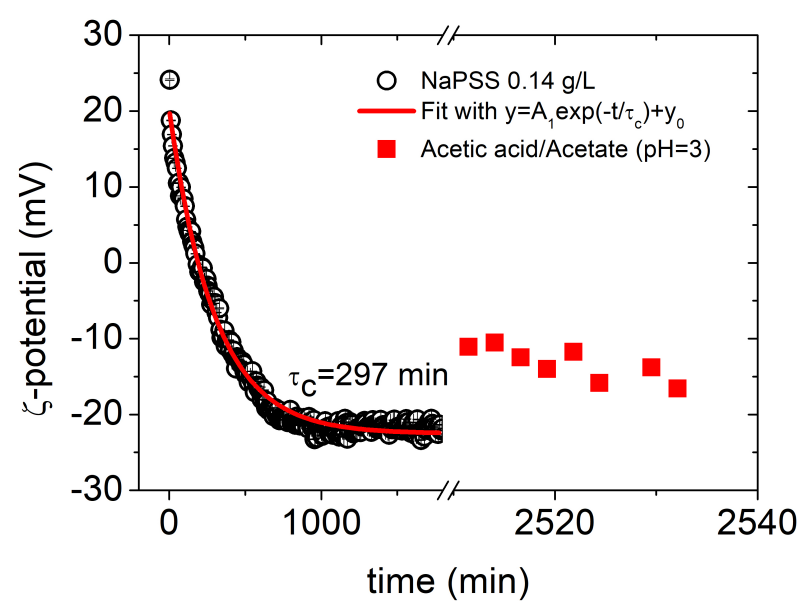

Figure 3. $\zeta$-potential evolution with time for AAOs measured in NaPSS aqueous solution (pure water at $\mathrm{pH}=6.5$ ) at $\mathrm{C}=0.14$ $\mathrm{g} / \mathrm{L}$ (black circles) followed by a washing in acetic acid/Acetate buffer solution at $\mathrm{pH}=3$ (red squares). The red continuous line corresponds to the best fit with a simple exponential.

We will now focus on the case of negative AAO. For direct comparison with a positive surface, we work under the same neutral $\mathrm{pH}$ condition. Fig. 4 presents the AAO $\zeta$ potential evolution with time according to a given sequence of buffer solutions. First, in region I, the AAO is immersed in pure water $(\mathrm{pH}=6.5)$ and the $\zeta$-potential value is positive, as expected, and around $+15 \mathrm{mV}$. Then, we immersed in a phosphate buffer solution at $\mathrm{pH}=6.1$ (region II) and the $\zeta$-potential was found to reverse to a negative value (around $-15 \mathrm{mV}$ ). As mentioned previously phosphate ions adsorb at the surface and the AAO becomes negatively charged. In region III, the negatively charged AAO is immersed in a NaPSS solution prepared in phosphate buffer at $\mathrm{pH}=6.1\left(\mathrm{C}_{\mathrm{PE}}=1.4 \mathrm{~g} / \mathrm{L}\right)$. The $\zeta$-potential remains unchanged (around $-15 \mathrm{mV}$ ) but we still cannot conclude whether PE adsorption took place or not. The PE might be competing with phosphate ions for adsorption. Finally, the AAO is immersed in a acetic/acetate solution at $\mathrm{pH}=4$ (region IV). If PE chain were adsorbed, the $\zeta$ potential would not vary (as in Fig. 3). On the contrary, if the $\zeta$-potential reversed to a positive value, then only phosphate ions would be adsorbed at the surface. One can see that the $\zeta$-potential values came back to $+15 \mathrm{mV}$ indicating that the PE was not adsorbed and the phosphate ions were washed away from the surface. By combining the results obtained for positive and negative AAOs, we can conclude that the NaPSS adsorption is mainly electrostatic (occurs only on positive surfaces) and is irreversible.

The experimental determination of the electrical surface properties of nanoporous materials is challenging. Here we show that TSPM is an efficient technique to:

i) determine the $\zeta$-potential of a model porous system (AAO) where the surface charge can be tuned to positive or negative values depending on the nature of the buffer solution.

ii) follow the $\zeta$-potential evolution when a PE diffuses inside. We show that, on a positive surface, the PE adsorbs irreversibly, while it does not when the surface is negative- ly charged, indicating the electrostatic nature of the PE adsorption.

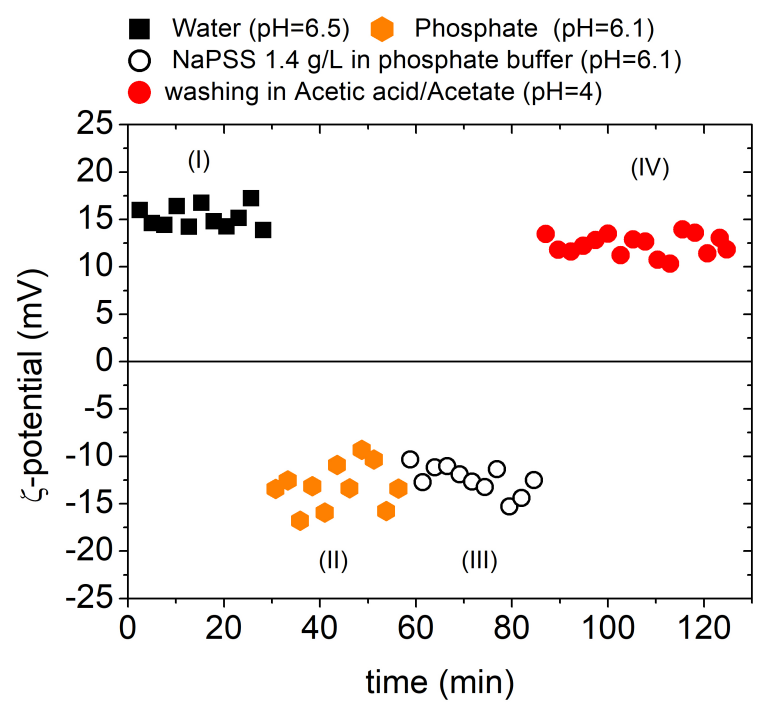

Figure 4. AAO $\zeta$-potential evolution with time throughout the following sequence of immersions: water at $\mathrm{pH}=6.5$ (black squares), phosphate buffer at $\mathrm{pH}=6.1$ (orange hexagons), NaPSS at $1.4 \mathrm{~g} / \mathrm{L}$ in phosphate buffer solution at $\mathrm{pH}=6.1$ (black circles), acetic acid/acetate at $\mathrm{pH}=4$ (red circles).

In the context of experimental limitations to investigate PE behavior on concave surfaces, which are expected to differ from convex ones according to recent theoretical work ${ }^{31}$, this study shows that TSPM is suitable to extract quantitative information regarding PE adsorption. It should further enable us to highlight the role of several parameters such as nanochannel size, ionic strength, PE nature, molecular weight and concentration... in the adsorption and desorption processes.

\section{ASSOCIATED CONTENT}

\section{Supporting Information}

The Supporting Information is available free of charge on the ACS Publications website:

Experimental details (SEM section view, flow measurements and TSPMs) and complementary $\zeta$-potential measurements.

\section{AUTHOR INFORMATION}

\section{Corresponding Author}

*(NJ). nicolas.jouault@sorbonne-universite.fr

\section{Author Contributions}

The manuscript was written through contributions of all authors.

\section{ACKNOWLEDGMENT}

The authors thank the Institut des Matériaux de Paris Centre (IMPC FR2482) for servicing FEGSEM and EDX instrumentation and Sorbonne Université, CNRS and C'Nano projects of the Région Ile-de-France for his funding and we thank David Montero for his help during the SEM characterization. We thank Natalie Malikova (PHENIX) for her constructive reading and comments of the manuscript. 


\section{REFERENCES}

1. Muthukumar, M. 50th anniversary perspective: A perspective on polyelectrolyte solutions. Macromolecules 2017, 50 (24), 9528-9560.

2. Dobrynin, A. V.; Colby, R. H.; Rubinstein, M. Scaling theory of polyelectrolyte solutions. Macromolecules 1995, 28 (6), 1859-1871.

3. Park, P. J.; Chun, M.-S.; Kim, J.-J. Partitioning and conformational behavior of polyelectrolytes confined in a cylindrical pore. Macromolecules 2000, 33 (23), 8850-8857.

4. Armstrong, J. A.; Bernal, E. E. L. n.; Yaroshchuk, A.; Bruening, M. L. Separation of ions using polyelectrolyte-modified nanoporous track-etched membranes. Langmuir 2013, 29 (32), 10287-10296.

5. $\quad$ Dotzauer, D. M.; Dai, J.; Sun, L.; Bruening, M. L. Catalytic Membranes Prepared Using Layer-by-Layer Adsorption of Polyelectrolyte/Metal Nanoparticle Films in Porous Supports. Nano Letters 2006, 6 (10), 2268-2272.

6. Ali, M.; Yameen, B.; Cervera, J.; Ramírez, P.; Neumann, R.; Ensinger, W.; Knoll, W.; Azzaroni, O. Layer-by-Layer Assembly of Polyelectrolytes into Ionic Current Rectifying Solid-State Nanopores: Insights from Theory and Experiment. J. Am. Chem. Soc 2010, 132 (24), 8338-8348.

7. Gilbert, E. P.; Auvray, L.; Lal, J. Structure of polyelectrolyte chains confined in nanoporous glass. Macromolecules 2001, 34 (14), 4942-4948.

8. $\quad$ Oukhaled, G.; Bacri, L.; Mathe, J.; Pelta, J.; Auvray, L. Effect of screening on the transport of polyelectrolytes through nanopores. Epl 2008, 82 (4).

9. Deen, W. M.; Smith, F. G. Hindered diffusion of synthetic polyelectrolytes in charged microporous membranes. Journal of Membrane Science 1982, 12 (2), 217-237.

10. Lazzara, T. D.; Lau, K. H. A.; Abou-Kandil, A. I.; Caminade, A.-M.; Majoral, J.-P.; Knoll, W. Polyelectrolyte Layer-by-Layer Deposition in Cylindrical Nanopores. ACS Nano 2010, 4 (7), 39093920.

11. Christoulaki, A.; Chennevière, A.; Dubois, E.; Jouault, N. Duplex nanoporous alumina and polyelectrolyte adsorption: more insights from a combined neutron reflectivity and electron microscopy study. Nanoscale 2019, 11 (5), 2148-2152.

12. Doane, T. L.; Chuang, C. H.; Hill, R. J.; Burda, C. Nanoparticle zeta-potentials. Accounts of Chemical Research 2012, 45 (3), 317-326.

13. Lucas, I. T.; Durand-Vidal, S.; Dubois, E.; Chevalet, J.; Turq, P. Surface Charge Density of Maghemite Nanoparticles: Role of Electrostatics in the Proton Exchange. The Journal of Physical Chemistry C 2007, 111 (50), 18568-18576.

14. Bluhm, E. A.; Bauer, E.; Chamberlin, R. M.; Abney, K. D.; Young, J. S.; Jarvinen, G. D. Surface Effects on Cation Transport across Porous Alumina Membranes. Langmuir 1999, 15 (25), 8668-8672.

15. Nyström, M.; Lindström, M.; Matthiasson, E. Streaming potential as a tool in the characterization of ultrafiltration membranes. Colloids and Surfaces 1989, 36 (3), 297-312.

16. Hernandez, A.; Martinez, F.; Martín, A.; Prádanos, P. Porous Structure and Surface Charge Density on the Walls of Microporous Alumina Membranes. Journal of Colloid and Interface Science 1995, 173 (2), 284-296.

17. Winkler, B. H.; Baltus, R. E. Modification of the surface characteristics of anodic alumina membranes using sol-gel precursor chemistry. Journal of Membrane Science 2003, 226 (1), 7584.

18. Pedimonte, B. J.; Moest, T.; Luxbacher, T.; von Wilmowsky, C.; Fey, T.; Schlegel, K. A.; Greil, P. Morphological zetapotential variation of nanoporous anodic alumina layers and cell adherence. Acta biomaterialia 2014, 10 (2), 968-974.
19. Burns, D. B.; Zydney, A. L. Buffer effects on the zeta potential of ultrafiltration membranes. Journal of membrane science 2000, 172 (1-2), 39-48.

20. Yanagishita, T.; Masuda, H. High-Throughput Fabrication Process for Highly Ordered Through-Hole Porous Alumina Membranes Using Two-Layer Anodization. Electrochimica Acta 2015, 184, 80-85.

21. Szymczyk, A.; Labbez, C.; Fievet, P.; Aoubiza, B.; Simon, C. Streaming potential through multilayer membranes. AIChE journal 2001, 47 (10), 2349-2358.

22. Kostaras, C.; Dellis, S.; Christoulaki, A.; Anastassopoulos, D. L.; Spiliopoulos, N.; Vradis, A.; Toprakcioglu, C.; Priftis, G. D. Flow through polydisperse pores in an anodic alumina membrane: A new method to measure the mean pore diameter. Journal of Applied Physics 2018, 124 (20), 204307.

23. Delgado, Á. V., Interfacial electrokinetics and electrophoresis. CRC Press: 2001; Vol. 106.

24. Delgado, A. V.; González-Caballero, F.; Hunter, R. J.; Koopal, L. K.; Lyklema, J. Measurement and interpretation of electrokinetic phenomena. Journal of Colloid and Interface Science 2007, 309 (2), 194-224.

25. Szymczyk, A.; Fievet, P.; Aoubiza, B.; Simon, C.; Pagetti, J. An application of the space charge model to the electrolyte conductivity inside a charged microporous membrane. Journal of Membrane Science 1999, 161 (1), 275-285.

26. Kosmulski, M., Chemical properties of material surfaces. CRC press: 2001.

27. Franks, G. V.; Gan, Y. Charging behavior at the aluminawater interface and implications for ceramic processing. Journal of the American Ceramic Society 2007, 90 (11), 3373-3388.

28. Sukhishvili, S. A.; Granick, S. Kinetic regimes of polyelectrolyte exchange between the adsorbed state and free solution. The Journal of chemical physics 1998, 109 (16), 6869-6878.

29. Duval, J. F. L.; Zimmermann, R.; Cordeiro, A. L.; Rein, N.; Werner, C. Electrokinetics of Diffuse Soft Interfaces. IV. Analysis of Streaming Current Measurements at Thermoresponsive Thin Films. Langmuir 2009, 25 (18), 10691-10703.

30. Zimmermann, R.; Dukhin, S. S.; Werner, C.; Duval, J. F. L. On the use of electrokinetics for unraveling charging and structure of soft planar polymer films. Current Opinion in Colloid \& Interface Science 2013, 18 (2), 83-92.

31. Gilles, F. M.; Boubeta, F. M.; Azzaroni, O.; Szleifer, I.; Tagliazucchi, M. Modulation of Polyelectrolyte Adsorption on Nanoparticles and Nanochannels by Surface Curvature. The Journal of Physical Chemistry C 2018, 122 (12), 6669-6677. 